\title{
Die zeitliche Ausbreitung von Potentialen in der Elektrodynamik
}

\author{
Von Walter Kaiser
}

\section{Einleitung}

Für eine die Gegensätze betonende Darstellung der Geschichte der Elektrodynamik haben sich seit langem die Begriffe «Fernwirkungstheorie» und «Nahwirkungstheorie» oder «Potentialtheorie» und «Feldtheorie» als griffige Vokabeln eingebürgert. Mit Selbstverständlichkeit wird vorausgesetzt, daß den Fernwirkungstheorien die Annahme instantaner, ohne Zeitverzug wirkender Fernkräfte zwischen elektrischen Ladungen und zwischen elektrischen Strömen zugrunde liegt. Die mathematische Beschreibung der elektrodynamischen Erscheinungen durch Kraftgesetze, die dem Newtonschen Gravitationsgesetz nachgebildet waren, schien keine andere Interpretation zuzulassen. Und in formaler Hinsicht ist dem zunächst kaum etwas entgegenzusetzen.

Aber ebensowenig wie Newton der instantan in die Ferne wirkenden Gravitationskraft physikalische Realität zugeschrieben hatte ${ }^{1}$, wollte etwa Ampère sein Kraftgesetz für die Wechselwirkung von Strömen als wirklich kausale Beschreibung betrachten. Angeregt durch Fresnels Theorie des quasi-elastischen Lichtäthers, formte sich Ampère qualitative physikalische Vorstellungen über einen einheitlichen Äther, der als Trägermedium für die Fortpflanzung von Lichtschwingungen und elektromagnetischen Kräften verantwortlich sein sollte ${ }^{2}$. Sehr deutlich zeigen sich auch die Bemühungen, den Mechanismus der Ausbreitung elektromagnetischer Kräfte in die theoretischen Betrachtungen einzubeziehen, bei Gauß. In einem bereits im 19. Jahrhundert vielzitierten Brief schrieb er 1845 sn Wilhelm Weber:

«Ich würde ohne Zweifel meine Untersuchungen [zur Elektrodynamik von 1834-1836] längst bekannt gemacht haben, hätte nicht zu der Zeit, wo ich sie abbrach, das gefehlt, was ich wie den eigentlichen Schlussstein betrachtet hatte

Nil actum reputans si quid superesset agendum

nemlich die Ableitung der Zusatzkräfte (die zu der gegenseitigen Wirkung ruhender Electricitätstheile noch hinzukommen, wenn sie in gegenseitiger Bewegung sind) aus der nicht instantaneen, sondern (auf ähnliche Weise wie beim Licht) in der Zeit sich fortpflanzenden Wirkung. Mir hatte dies damals nicht gelingen wollen; ich verliess aber so viel ich mich erinnere die Untersuchung damals doch nicht ganz ohne Hoffnung, dass dies später vielleicht gelingen könnte, obwohl - erinnere ich mich recht - mit der subjectiven Überzeugung, dass es vorher nöthig sei, sich von der Art, wie die Fortpflanzung geschieht, eine construirbare Vorstellung zu machen [Hervorhebungen von Gauß].» ${ }^{3}$ 
Webers Antwort war recht knapp, im Grunde aber zustimmend: «Gewiß wäre die Erklärung aus einer allmählichen Fortpflanzung der Wirkung die schönste Lösung des Rätsels. » ${ }^{4}$ Auch im Zusammenhang mit der Formulierung des berühmten Grundgesetzes, das die Phänomene der stationären Elektrodynamik auf die Wechselwirkung geladener Teilchen zurückführte, finden sich bei Weber Überlegungen, die eine Übertragung elektrodynamischer Kräfte durch ein Zwischenmedium zum Inhalt haben ${ }^{5}$. Entscheidend für solche Vorstellungen war wohl einerseits die Kenntnis der zeitlichen Ausbreitung von Licht und andererseits die ungeklärte Frage nach der Natur des Lichts. Nach 1845, also nach der Entdeckung des Faradayeffekts, lag hier ein wichtiges experimentelles Indiz für eine enge Beziehung zwischen «Lichtäther» und «elektrischem Äther» vor ${ }^{6}$. Obwohl Weber außerdem 1855 (bei der Bestimmung des Verhältnisses von elektrostatisch gemessener Ladungseinheit zu elektrodynamisch gemessener Ladungseinheit) auf eine Konstante von der Größenordnung der Lichtgeschwindigkeit stie $\beta^{7}$, vermochte er seine qualitativen Überlegungen nicht in mathematische Formulierungen umzusetzen. Im Gegenteil, als 1857 Kirchhoff für die Fortpflanzungsgeschwindigkeit der «Elektrizität» in Drähten die Lichtgeschwindigkeit erhielt, äußerte sich Weber sehr skeptisch über einen Zusammenhang der «Lehren » vom Licht und von der Elektrizität ${ }^{8}$.

Etwa gleichzeitig hatte Maxwell begonnen, die suggestiven gedanklichen Modelle Faradays, insbesondere die Feldvorstellung, in mathematische Form zu bringen. Während die Kraftgesetze bzw. die Potentialgesetze der Fernwirkungstheorien die elektrodynamischen Phänomene auf die instantane Wechselwirkung entfernter Stromelemente oder Ladungen zurückführten, wurde nun anhand der Maxwellschen Differentialgleichungen eine lokale Beschreibung der Wirkung von Strömen und Ladungen gegeben, wobei dem Feldbegriff die Vorstellung zugrunde lag, daß sich materie- und äthererfüllter Raum (also auch das Vakuum) in kontinuierlicher Weise unter dem Einfluß elektrischer und magnetischer Kräfte verändern. Insbesondere tragen demnach zeitlich wechselnde elektrische Felder durch die zeitlich wechselnde Polarisation eines Dielektrikums zum Gesamtstrom eines elektrodynamischen Systems bei. Eine der wesentlichen Folgerungen aus den Maxwellschen Gleichungen war bekanntlich die Ableitung von Wellengleichungen für die Ausbreitung freier elektromagnetischer Felder und damit das Konzept einer elektromagnetischen Lichttheorie.

Andererseits gab es schon früh Versuche, im Anschluß an die qualitativen Überlegungen von Ampère und Gauß auch die mathematischen Formulierungen der Fernwirkungstheorien so zu ergänzen, daß sie die zeitliche Ausbreitung elektrischer Wirkungen mit umfaßten. Neben der Maxwellschen Feldtheorie, die eine 
lokale raumzeitliche Beschreibung der Ausbreitung elektrischer Kräfte bot, entstand hier das Konzept des retardierten Potentials, das die augenblicklichen elektromagnetischen Wirkungen auf eine zeitlich zurückliegende Ursache, auf eine Konstellation entfernter Ladungen und Ströme zurückführte. Die Retardierung $\frac{\left|\mathfrak{r}-\mathbf{r}^{\prime}\right|}{\mathrm{c}}$ entspricht dabei gerade der Zeit, die eine Wirkung mit der Ausbreitungsgeschwindigkeit c braucht, um vom Quellpunkt $\mathfrak{r}^{\prime}$ zum Aufpunkt $\mathfrak{r}$ zu gelangen. Damit zeichnete sich bereits zu dem Zeitpunkt, an dem eine divergente Entwicklung von Fernwirkungstheorien und Nahwirkungstheorien hätte beginnen können (und im Bewußtsein vieler Physiker des 19. Jahrhunderts auch wirklich begonnen hat) eine entscheidende Verringerung der Gegensätze, eine Tendenz zu einer einheitlichen Beschreibungsmöglichkeit durch Felder und retardierte Potentiale ab.

Bernhard Riemann (1858), Ludvig Valentin Lorenz (1867), Carl Neumann (1868) und Enrico Betti (1868) haben hier die ersten wichtigen Beiträge geleistet. Eng mit den Arbeiten von Riemann und Lorenz verknüpft ist auch der Versuch von Heinrich Hertz (1884), den nun aufgebrochenen Graben zwischen den älteren Theorien der Elektrodynamik und der Maxwellschen Theorie zu überbrücken. Diese historisch und inhaltlich eng zusammenhängenden Arbeiten, die den Aspekt der zeitlichen Ausbreitung von Potentialen in die Diskussion der elektrodynamischen Theorien brachten, sollen in der Folge etwas näher betrachtet werden.

\section{Bernhard Riemanns «Beitrag zur Elektrodynamik»}

Die nur wenige Seiten umfassende Arbeit von Bernhard Riemann wurde 1858 der Königlichen Gesellschaft der Wissenschaften in Göttingen übergeben, vor der Publikation aber wieder zurückgezogen ${ }^{9}$ und erst 1867 posthum in den Annalen der Physik veröffentlicht ${ }^{10}$. Obwohl der Titel der Arbeit schlicht «Ein Beitrag zur Elektrodynamik» lautet, verraten die einleitenden Bemerkungen, welche Motive dieser Untersuchung zugrunde lagen.

«Der Königlichen Societät erlaube ich mir eine Bemerkung mitzutheilen, welche die Theorie der Elektricität und des Magnetismus mit der des Lichts und der strahlenden Wärme in einen nahen Zusammenhang bringt. Ich habe gefunden, dass die elektrodynamischen Wirkungen galvanischer Ströme sich erklären lassen, wenn man annimmt, dass die Wirkung einer elektrischen Masse auf die übrigen nicht momentan geschieht, sondern sich mit einer constanten (der Lichtgeschwindigkeit innerhalb der Grenzen der Beobachtungsfehler gleichen) Geschwindigkeit zu ihnen fortpflanzt. Die Differentialgleichung für die Fortpflanzung der elektrischen Kraft wird bei dieser Annahme dieselbe, wie für die Fortpflanzung des Lichts und der strahlenden Wärme. » ${ }^{11}$ 
Riemann, der durch Wilhelm Weber in das physikalische Denken eingeführt worden war, hat offenbar bei der Formulierung dieser Zusammenhänge sehr mit sich gerungen. Gegenüber der vorsichtigen Formulierung in der gedruckten Abhandlung, nach der die Lichtausbreitung und die Ausbreitung elektrischer Kräfte in formaler Hinsicht verglichen werden, finden sich unter den Entwürfen zum Manuskript auch Fassungen, in denen geradezu von einer substantiellen Identität von Licht und elektrischen Kräften die Rede ist:

«Es pflanzt sich also die electrische Kraft nach demselben Gesetze fort, wie Licht und strahlende Wärme, und es ist nicht zu zweifeln, daß electrische Kraft, Licht und strahlende Wärme durch denselben Prozeß fortgepflanzt werden (und nur verschiedene Äußerungen derselben Ursache sind [Im Ms. wieder gestrichen]). ${ }^{12}$

Dies stimmt mit einer anderen Feststellung überein, in der von «einem Medium » die Rede ist ${ }^{13}$, in dem sich Licht und elektrische Wirkungen fortpflanzen. Die Streichung im Manuskript zeigt jedoch, daß diese weitreichende und vielleicht noch etwas spekulative Aussage für die Veröffentlichung nicht verantwortet werden konnte.

Dabei waren die Überlegungen Riemanns bereits an einem entscheidenden Punkt der Formulierung einer elektromagnetischen Lichttheorie angelangt. Riemann nahm an, daß die Differentialgleichung der Elektrostatik

$$
\Delta \mathrm{U}(\mathfrak{r})=4 \pi \varrho(\mathfrak{r})
$$

zu ersetzen ist durch die heute sogenannte inhomogene Wellengleichung für das skalare Potential U ${ }^{14}$. Die Wellengleichung

$$
\Delta \mathrm{U}(\mathfrak{r})-\frac{1}{\mathrm{c}^{2}} \frac{\partial^{2} \mathrm{U}(\mathfrak{r})}{\partial \mathrm{t}^{2}}=4 \pi \varrho(\mathfrak{r})
$$

beschreibt die zeitliche Ausbreitung elektrischer Wirkungen, wobei hier $\varrho(\mathfrak{r})$ die räumliche Ladungsdichte und c die Lichtgeschwindigkeit bedeuten.

Obwohl sich in den Manuskripten Riemanns Versuche finden ${ }^{15}$, die inhomogene Wellengleichung für das skalare Potential aus einem Wirkungsprinzip herzuleiten, wird diese Differentialgleichung im Grunde postuliert. Auch die Lösung wird nur im Ansatz durchgeführt. Riemann formulierte zwar ein retardiertes Potential, allerdings nicht für eine beliebige Ladungsverteilung $\varrho(\mathfrak{r})$, wie sie auch der Wellengleichung zugrunde liegt, sondern von vornherein für eine punktförmige Gesamtladung $-f(t)$. 
Anstatt des retardierten Potentials

$$
\mathrm{U}(\mathrm{r})=-\int \frac{\varrho\left(\mathbf{r}^{\prime}, \mathbf{t}-\frac{\left|\mathbf{r}-\mathbf{r}^{\prime}\right|}{\mathbf{c}}\right)}{\left|\mathbf{r}-\mathbf{r}^{\prime}\right|} \mathbf{d}^{3} \mathrm{r}^{\prime}
$$

findet sich bei Riemann der Ausdruck

$$
\mathrm{U}(\mathfrak{r})=\frac{\mathbf{f}\left(\mathbf{t}-\frac{\left|\mathfrak{r}-\mathbf{r}^{\prime}\right|}{\mathbf{c}}\right)}{\left|\mathbf{r}-\mathbf{r}^{\prime}\right|} .
$$

Ausgehend von der Idee der Retardierung eines Potentials, versuchte Riemann nun, ein dem Weberschen Gesetz vergleichbares Wechselwirkungsgesetz für bewegte Ladungen abzuleiten. - Ziel war ja die Erklärung der «elektrodynamischen Wirkungen galvanischer Ströme » aus einer (der Lichtausbreitung vergleichbaren) endlichen Ausbreitung elektrischer Wirkungen. Die mathematischen Umformungen Riemanns sind jedoch fragwürdig, und bereits Rudolf Clausius ${ }^{16}$ hat diese Arbeit bald nach ihrem Erscheinen einer scharfen Kritik unterzogen.

Was aber diesen Versuch aus heutiger Sicht trotzdem interessant macht, ist die Tatsache, da $\beta$ er den Grundgedanken der Liénard-Wiechert-Potentiale für beliebig bewegte Punktladungen vorwegnimmt, indem der Abstand zweier wechselwirkender geladener Teilchen als Funktion der retardierten Zeit aufgefaßt wird $^{17}$. Die Unzulänglichkeit der Lösung der inhomogenen Wellengleichung zeigt sich allerdings auch am «Liénard-Wiechert»-Ansatz von Riemann. Hier wird nicht über eine Ladungsverteilung unter Berücksichtigung der verschiedenen retardierten Zeiten integriert, sondern sofort der Abstand zweier Punktladungen als Funktion der retardierten Zeit angeschrieben. Das Wechselwirkungspotential $\mathrm{P}$ zweier Punktladungen $\varepsilon$ und $\varepsilon^{\prime}$ lautet bei Riemann also

$$
\mathrm{P}=-\frac{\varepsilon \varepsilon^{\prime}}{\left|\mathrm{r}-\mathrm{r}^{\prime}\right|\left(\mathrm{t}-\frac{\left|\mathbf{r}-\mathbf{r}^{\prime}\right|}{\mathrm{c}} ; \mathrm{t}\right)} .
$$

Alfred Liénard ${ }^{18}$ und Emil Wiechert ${ }^{19}$ haben dann 1898 bzw. 1900 die retardierten Potentiale für beliebig bewegte Punktladungen exakt angegeben. Demnach ist zum Beispiel das skalare Potential $\varphi$ einer mit der Geschwindigkeit $\mathfrak{b}$ bewegten punktförmigen Ladung e

$$
\varphi(\mathfrak{r})=\frac{\mathbf{e}}{\left[\left|\mathfrak{r}-\mathbf{r}^{\prime}\right|-\frac{\left(\mathfrak{r}-\mathbf{r}^{\prime}\right) \cdot \mathfrak{v}}{\mathbf{c}}\right]} \mathbf{t}^{\prime}=\mathbf{t}-\frac{\left|\mathbf{r}-\mathbf{r}^{\prime}\right|}{\mathbf{c}} .
$$

Emil Wiechert lehnte sich dabei eng an Webers Elektrodynamik geladener Teilchen an, die durch Experimente mit Kathodenstrahlen an empirischer Bedeutung 
gewonnen hatte. Die eigentliche Ableitung der später sogenannten LiénardWiechert-Potentiale war dann expressis verbis eine Kritik der Arbeit Riemanns von $1858^{20}$.

Ergänzend ist zu bemerken, daß Riemann bei Betrachtungen über die Potentiale gleichförmig bewegter Punktladungen spätestens 1861 die heute als LorentzKonvention oder Lorentz-Eichung bekannte Beziehung

$$
\operatorname{div} \mathfrak{U}+\frac{1}{\mathrm{c}} \frac{\partial \varphi}{\partial \mathrm{t}}=0
$$

angegeben hat ${ }^{21}$. Mit der Lorentz-Konvention, mit der als Erhaltungssatz ebenfalls in seinen Vorlesungen formulierten Kontinuitätsgleichung ${ }^{22}$ und mit der inhomogenen Wellengleichung für das skalare Potential hat also Riemann 1858 und 1861 einige wichtige Teile des Gleichungssystems der klassischen Elektrodynamik angegeben. Allerdings waren diese Gleichungen ohne volle Berücksichtigung des Vektorpotentials bzw. des magnetischen Feldes letzten Endes doch zur fragmentarischen, unzulänglichen Theorie gestempelt.

\section{Ludvig Valentin Lorenz und das retardierte Potential}

Johann Christian Poggendorff, der langjährige Herausgeber der Annalen der Physik, hat sicher nicht ohne sorgfältige U̇berlegung der genannten Arbeit von Riemann unmittelbar die Abhandlung «Über die Identität der Schwingungen des Lichts mit den elektrischen Strömen ${ }^{23}$ von Lorenz folgen lassen. In zwei wesentlichen Punkten stimmen die beiden Arbeiten überein: in der Annahme inhomogener Wellengleichungen bzw. retardierter Potentiale und in dem Bestreben, aus der Elektrodynamik heraus eine theoretische Erklärung für die Phänomene des Lichts zu geben. Aufschlußreich ist auch bei Lorenz die Einleitung. Er äußert sich hier entgegen den empiristischen Tendenzen seiner Zeitgenossen geradezu im Stil der romantischen Physiker über den Zusammenhang der Naturkräfte :

«Bekanntlich ist es der Wissenschaft unseres Jahrhunderts gelungen, so viele Beziehungen zwischen den verschiedenen Kräften, zwischen Elektricität und Magnetismus, zwischen Wärme, Licht, molecularen und chemischen Kräften, nachzuweisen, daß man mit einer gewissen Nothwendigkeit dahin geführt wird, sie alle als Äußerungen einer und derselben Kraft zu betrachten, die nach den Umständen unter verschiedenen Formen auftritt [Hervorhebung von Lorenz]. ${ }^{24}$

An anderen Stellen seiner Arbeit erweist sich ebenfalls, wie sehr Lorenz in den gedanklichen Kontext der Entwicklung der Elektrodynamik integriert war. Nicht verwunderlich ist, daß er als Däne die von Oersted entdeckte Wechsel- 
wirkung von Strömen und Magneten als Beispiel für den Nachweis dieser Zusammenhänge zwischen den Kräften zitiert ${ }^{25}$. Ganz im Einklang mit der immer wieder feststellbaren Konvergenz der Entwicklung in der Elektrodynamik und der Verflechtung der Argumentation verweist Lorenz auch auf die Anregungen von Ampère und Faraday:

«Hieraus ... würde ... resultiren ..., daß jede Wirkung der Elektricität und der elektrischen Ströme in der Wirklichkeit nur von dem elektrischen Zustande der nächst umgebenden Elemente abhängt in der Weise, wie es die Differentialgleichungen (B) [gemeint ist die Gleichung (13)] angeben. Dies ist bekanntlich schon von ... Ampère angedeutet und von mehreren Physikern, namentlich von Faraday, verfochten worden [Hervorhebungen von Lorenz]. ${ }^{26}$

Was ihre Entstehung betrifft, ist die Arbeit von Lorenz offenbar von Maxwells elektromagnetischer Lichttheorie unabhängig, obwohl sie 1867, also zwei Jahre nach Maxwells "A dynamical theory of the electromagnetic field" veröffentlicht wurde. 1868 und 1873 hat Maxwell, dem die formale Ähnlichkeit seiner Theorie mit den Ansätzen von Riemann und Lorenz nicht entgangen war, auf die verschiedenen Erscheinungsdaten im Sinne eines Prioritätsanspruches hingewie$\operatorname{sen}^{27}$.

Der Ausgangspunkt von Lorenz war eine von Kirchhoff auf der Basis der Induktionsgesetze von Weber und Neumann aufgestellte Gleichung für die Stromdichte $\dot{i}^{28}$ :

$$
\dot{i}=-\sigma\left(\operatorname{grad} \varphi+\frac{1}{c} \frac{\partial \mathfrak{U}}{\partial t}\right)
$$

in der das Ohmsche Gesetz und das Induktionsgesetz zusammengefaßt sind ( $\sigma$ ist die Leitfähigkeit, c die Lichtgeschwindigkeit). Lorenz stellt sich nun vor, daß die ursprünglich in Gleichung (8) enthaltenen Potentiale $\varphi$ und $\mathfrak{A}$ nur die ersten Glieder von Reihenentwicklungen der eigentlichen, retardierten Potentiale sind. Zum Beispiel wäre also das vollständige skalare Potential so zu schreiben:

$$
\begin{gathered}
\varphi(\mathfrak{r}, \mathrm{t})=\int \frac{\varrho\left(\mathrm{r}^{\prime}, \mathrm{t}\right)}{\left|\mathrm{r}-\mathrm{r}^{\prime}\right|} \mathrm{d}^{3} \mathrm{r}^{\prime}-\frac{1}{\mathrm{c}} \frac{\partial}{\partial \mathrm{t}} \int \varrho\left(\mathrm{r}^{\prime}, \mathrm{t}\right) \mathrm{d}^{3} \mathrm{r}^{\prime}+\frac{1}{2 \mathrm{c}^{2}} \frac{\partial^{2}}{\partial \mathrm{t}^{2}} \int\left|\mathrm{r}-\mathrm{r}^{\prime}\right| \varrho\left(\mathrm{r}^{\prime}, \mathrm{t}\right) \mathrm{d}^{3} \mathrm{r}^{\prime} \ldots \\
\text { oder kürzer } \\
\varphi(\mathrm{r}, \mathrm{t})=\int \frac{\varrho\left(\mathrm{r}^{\prime}, \mathrm{t}-\frac{\left|\mathrm{r}-\mathrm{r}^{\prime}\right|}{\mathrm{c}}\right)}{\left|\mathrm{r}-\mathrm{r}^{\prime}\right|} \mathrm{d}^{3} \mathrm{r}^{\prime}
\end{gathered}
$$

Dabei argumentierte Lorenz folgendermaßen :

«Es ist nun sogleich einleuchtend, daß die Gleichungen (1) [Hier Gleichung (8); der Plural bezieht sich auf die Komponentenschreibweise bei Lorenz], die in völlig empirischer 
Weise hergeleitet sind, nicht nothwendig der exacte Ausdruck des wirklichen Gesetzes sind, und es wird immer erlaubt seyn, mehrere Glieder zuzufügen oder den Gleichungen eine andere Form zu geben, so lange nur diese Änderungen keinen merklichen Einfluß auf die Resultate, die durch Versuche constatirt sind, bekommen können. Wir werden daher damit anfangen, die zwei Glieder auf der rechten Seite der Gleichungen (1) [Hier Gleichung (8)] als die ersten Glieder einer Reihenentwickelung zu betrachten [Hervorhebung von Lorenz]. ${ }^{29}$

Im Zusammenhang mit einer 1861 veröffentlichten elastomechanischen Theorie, die die Ausbreitung von Störungen in einem unbegrenzten elastischen Medium zum Inhalt hatte, hatte Lorenz das retardierte Potential als Lösung einer inhomogenen Wellengleichung bereits angegeben ${ }^{30}$. Für seine Argumentation in der Elektrodynamik benutzte Lorenz die inhomogene Wellengleichung und das retardierte Potential zunächst in allgemeiner Form ${ }^{31}$ :

$$
\left(\Delta-\frac{1}{c^{2}} \frac{\partial^{2}}{\partial t^{2}}\right) \int \frac{f\left(r^{\prime}, t-\frac{\left|r-r^{\prime}\right|}{c}\right.}{\left|r-r^{\prime}\right|} d^{3} r^{\prime}=-4 \pi f(r, t) .
$$

Wenn man den Differentialoperator $\left(\Delta-\frac{1}{\mathrm{c}^{2}} \frac{\partial^{2}}{\partial \mathrm{t}^{2}}\right)$ bzw. $\square$ auf Gleichung (8) anwendet, ergibt sich

$$
\square \mathrm{i}=-\sigma\left(\operatorname{grad} \square \varphi+\frac{1}{\mathrm{c}} \frac{\partial}{\partial \mathrm{t}} \square \mathfrak{U}\right) \text {. }
$$

Mit div $\leftleftarrows=4 \pi \varrho$ und unter Berücksichtigung des Ohmschen Gesetzes $\dot{i}=\sigma(\xi$ (wo \& die elektrische Feldstärke ist) erhielt Lorenz

$$
\Delta \mathrm{i}-\frac{1}{\mathbf{c}^{2}} \frac{\partial^{2}}{\partial \mathbf{t}^{2}} \mathrm{i}=\operatorname{grad} \operatorname{div} \mathrm{i}+\frac{4 \pi \sigma}{\mathbf{c}^{2}} \frac{\partial}{\partial \mathbf{t}} \dot{\mathrm{i}}
$$

und schließlich

$$
\operatorname{rot} \operatorname{rot} \dot{i}+\frac{4 \pi \sigma}{\mathbf{c}^{2}} \frac{\partial}{\partial \mathbf{t}} \dot{\mathrm{i}}+\frac{1}{\mathrm{c}^{2}} \frac{\partial^{2}}{\partial \mathrm{t}^{2}} \dot{\mathrm{i}}=0
$$

Gleichung (14) ist die von Lorenz hergeleitete «Wellengleichung für Ströme». ${ }^{32}$ Die Behauptung von der «Identität der Schwingungen des Lichts mit den elektrischen Strömen » rührt nun daher, daß Lorenz ein formal ähnliches Ergebnis 1864 im Rahmen einer Lichttheorie des elastischen Lichtäthers erhalten hatte ${ }^{33}$. In diesem Zusammenhang ist noch zu bemerken, daß Lorenz nicht, wie hier geschehen, von vornherein die Geschwindigkeit $\mathrm{c}$ in Gleichung (8) und in Gleichung (11) mit der Lichtgeschwindigkeit identifiziert hat, sondern genau dann, als es darum ging, Gleichung (14) als Ausbreitungsgleichung für Licht zu interpretieren. 
Setzt man wie in der Theorie Maxwells einen frequenzunabhängigen Widerstand voraus und nimmt man weiter an, daß keine Ladungsdichte vorhanden ist, läßt sich Gleichung (12) mit Hilfe des Ohmschen Gesetzes in eine etwas anschaulichere Form bringen:

$$
\Delta E-\frac{1}{c^{2}} \frac{\partial^{2}}{\partial t^{2}} \xi-\frac{\partial}{\partial t} \frac{4 \pi \sigma}{c^{2}} \xi=0 .
$$

Damit wird deutlich, daß Lorenz eine Wellengleichung für den Fall eines über den ganzen Raum ausgedehnten homogenen leitenden Mediums abgeleitet hat. Physikalisch relevant ist etwa der Fall hoher Frequenzen, für den Gleichung (15) in die gewöhnliche homogene Wellengleichung übergeht, was ungedämpfte Ausbreitung, also Durchsichtigkeit bedeutet. Dasselbe gilt für einen Isolator. Für den Fall, daß die Absorption zum Tragen kommt, insbesondere bei guten Leitern, gilt die volle Gleichung (15), allerdings mit der bei der experimentellen Diskussion der Maxwellschen Theorie wichtig gewordenen Einschränkung, daß Gleichung (15) mit frequenzunabhängiger Leitfähigkeit die Metallreflexion nicht exakt beschreibt. Für den Fall niederer Frequenzen kann der Term mit doppelter Zeitableitung in Gleichung (15) vernachlässigt werden. Die verbleibende Gleichung liegt zusammen mit Randbedingungen der Theorie des Skineffektes zugrunde. Lorenz selbst hat Absorption und Durchsichtigkeit diskutiert, allerdings nur unter dem Aspekt der Leitfähigkeit ${ }^{34}$. Fehlte bei Riemann noch die inhomogene Wellengleichung für das Vektorpotential, liegt nun bei Ludvig Valentin Lorenz praktisch das vollständige Gleichungssystem der Elektrodynamik vor. Vollständig auch deshalb, weil Lorenz zusammen mit der Kontinuitätsgleichung und der inhomogenen Wellengleichung ebenfalls die (nach Hendrik Antoon Lorentz benannte) Lorentz-Eichung angegeben hatte ${ }^{35}$.

\section{Der Gedanke der Retardierung bei Enrico Betti und Carl Neumann}

Enrico Betti, der mit Riemann befreundet war, veröffentlichte 1868 im Anschluß an Riemanns posthume Arbeit von 1867 eine «Notiz über die Elektrodynamik». ${ }^{36}$ Eine weitere unmittelbare Folge war Carl Neumanns Abhandlung über «Die Principien der Elektrodynamik ${ }^{37}$ aus demselben Jahr. Obwohl beide Autoren Lorenz nicht ausdrücklich erwähnen, darf wohl angenommen werden, daß Lorenz' Arbeit von 1867, die in den Annalen der Physik unmittelbar auf die von Riemann folgte, mit dazu beigetragen hat, das Thema einer zeitlichen Ausbreitung von Potentialen aufzugreifen. Betti weist aber zu Beginn seiner Arbeit auf eine weitere wichtige Anregung hin ${ }^{38}$, auf den bereits erwähnten, seit 1867 gedruckt vorliegenden Brief von Gauß an Weber, in dem ja von einer «nicht instantaneen», 
«in der Zeit sich fortpflanzenden Wirkung» die Rede ist. Rudolf Clausius hat dann auch in seiner kritischen Würdigung die Arbeiten von Riemann, Betti und Carl Neumann als « von Gauß angeregte neue Auffassung der elektrodynamischen Erscheinungen » charakterisiert ${ }^{39}$.

Den historischen Verflechtungen entsprechen die inhaltlichen Zusammenhänge. Ein Aspekt der Arbeit von Riemann, und zwar die Ableitung eines Wechselwirkungsgesetzes für Ströme bzw. für bewegte Ladungen, steht auch im Mittelpunkt der Überlegungen von Betti. Ähnlich wie Riemann betrachtete Betti den Abstand zweier bewegter Ladungen als Funktion der retardierten Zeit. Er versuchte nun zu zeigen, daß eine Berücksichtigung des Retardierungseffektes bis zur zweiten Näherung mit einem Wechselwirkungsgesetz für quasi-stationäre Ströme vereinbar ist. Da Betti aber von zeitlich sich rasch ändernden Strömen ausging, deren Änderungsgeschwindigkeit die Lichtgeschwindigkeit sogar überstieg ${ }^{40}$, blieb seine Argumentation letztlich problematisch.

Interessant ist jedoch die gewählte Näherung, das heißt die Beschränkung des Retardierungseffektes auf die zweite Näherung. Auch Carl Neumann (der übrigens jeden Zusammenhang mit der Theorie des Lichts strikt ablehnte ${ }^{41}$ ) benutzte in seiner Schrift über «Die Principien der Elektrodynamik» das retardierte Potential bzw. dessen Reihenentwicklung nur bis zu Gliedern der Ordnung $\frac{\left|\mathfrak{r}-\mathbf{r}^{\prime}\right|^{2}}{\mathbf{c}^{2}} 42$. Wie bei Riemann und Betti war es dabei das Ziel, ein Wechselwirkungsgesetz für bewegte Ladungen, hier das Webersche Gesetz, abzuleiten.

Wir wissen heute, daß die Berücksichtigung des Retardierungseffektes bis zur zweiten Ordnung gerade der strahlungsfreien Näherung entspricht ${ }^{43}$. Allerdings hat sich gezeigt ${ }^{44}$, daß die Wechselwirkung bewegter geladener Teilchen in dieser Näherung nicht durch «Grundgesetze» wie das Webersche Gesetz, sondern durch das von Charles G. Darwin ${ }^{45}$ und Gregory Breit ${ }^{46} 1920$ bzw. 1929 aufgestellte Wechselwirkungspotential beschrieben wird. Erstaunlicherweise hatte jedoch bereits ein 1870 von Hermann Helmholtz postuliertes allgemeines Wechselwirkungspotential für Stromelemente ${ }^{47}$ die Struktur des Darwin-Breit-Potentials. Hier ging also der Effekt der Retardierung fast zufällig in die mathematische Formulierung ein. Trotzdem könnte man etwas überspitzt sagen, daß auch bei der Diskussion über die Wechselwirkungsgesetze für bewegte Ladungen, über die typischen Fernwirkungsgesetze also, gerade das Prinzip der instantanen Fernwirkung durchbrochen worden ist. 


\section{Die «Ableitung» einer Wellengleichung für Potentiale durch Heinrich Hertz}

Wie aus dem Tagebuch von Heinrich Hertz aus dem Jahre 1884 hervorgeht, ist die Abhandlung «Über die Beziehungen zwischen den Maxwellschen elektrodynamischen Grundgleichungen und den Grundgleichungen der gegnerischen Elektrodynamik $» 48$ aus vielfältigen Überlegungen zur Maxwellschen Theorie hervorgegangen. Es finden sich etwa folgende Eintragungen: «Elektromagnetische Lichttheorie nachgedacht.» «Den ganzen Tag Elektrodynamik gearbeitet.» «Abends tüchtig. Elektrodynamik nach Maxwell.»Und schließlich: «Morgens glückte die elektrodynamische Frage. ${ }^{49}$

Hertz' Untersuchung hatte jedoch zunächst das Ziel, mit den Begriffen und Argumentationsweisen der älteren Theorien der Elektrodynamik eine (inhomogene) Wellengleichung für Potentiale abzuleiten. Sehr deutlich ist hier der erwähnte historische Zusammenhang mit den Arbeiten von Bernhard Riemann und Ludvig Valentin Lorenz:

«Gleiche, res.[pektive] ganz ähnliche Gesetze für die Ausbreitung der Potentiale haben im Jahre 1858 Riemann und im Jahre 1867 Lorenz angenommen, in dem Wunsche, die optischen und elektrischen Erscheinungen unter gemeinsamen Gesichtspunkten zu vereinigen. Daß durch diese Gesetze den geltenden Kräften der Elektrodynamik neue Glieder hinzugefügt wurden, entschuldigten diese Forscher damit, daß diese Glieder zu klein seien ${ }^{50}$, als daß sie sich in den Experimenten hätten bemerkbar machen können. Wir sehen aber, daß die Hinzufügung dieser Glieder weit entfernt ist, einer Entschuldigung zu bedürfen, daß sie viel eher geboten ist, und daß das Fehlen dieser Glieder die Verletzung ganz allgemein angenommener Prinzipien involvieren müßte. ${ }^{51}$

Die wichtigsten allgemeinen Prinzipien, die nach Hertz auch den vor-maxwellschen Theorien zugrunde liegen, sind die Voraussetzung der «Einheit der magnetischen Kraft», die Hertz auf Ampère zurückführt ${ }^{52}$, und die «Einheit der elektrischen Kraft». Gemeint ist damit, daß es bei «magnetischen Kräften» gleichgültig ist, ob diese Kräfte von einem permanenten Magneten oder von einem stromdurchflossenen Leiter herrühren. Die «Einheit der elektrischen Kraft» beruht auf der Gleichwertigkeit der «elektrischen Kräfte», die von einer elektrischen Ladung ausgehen, und derjenigen, die von veränderlichen Strömen durch Induktion erzeugt werden. Dies bedeutet letztlich, daß magnetisches und elektrisches Feld unabhängig von ihrer «Erzeugungsweise im Raum» existieren ${ }^{53}$.

Hertz geht nun von einem System geschlossener elektrischer Ströme aus, das sich im Vakuum befindet. Als Beschreibung dieser Stromverteilung i betrachtet er das zugehörige Vektorpotential $\mathfrak{U}$

$$
\mathfrak{U}(\mathfrak{r})=\int \frac{i\left(\mathfrak{r}^{\prime}\right)}{\left|\mathfrak{r}-\mathbf{r}^{\prime}\right|} d^{3} \mathbf{r}^{\prime} .
$$


Dabei stellt $\mathfrak{A}(\mathfrak{r})$ die Lösung der Differentialgleichung $\Delta \mathfrak{A}(\mathfrak{r})=-4 \pi \dot{j}(\mathfrak{r})$ dar.

Der weiteren Argumentation liegt folgender Gedankenversuch zugrunde: In einem ringförmigen Magneten (in einem «Ring weichen Eisens») existiere ein zeitlich veränderliches Magnetfeld $\mathfrak{Z}$ (ein «magnetischer Strom»). Dieses veränderliche Magnetfeld induziere ein elektrisches Feld (ङ. «Einheit» der elektrischen Kraft bedeutet dann, daß sich die elektrische Wechselwirkung solcher veränderlicher Magnete im Prinzip, d.h. ohne Rücksicht auf Größenordnung und Isolierbarkeit des Effekts, wie die Wechselwirkung elektrischer Ladungen behandeln läßt ${ }^{54}$, ähnlich wie die Wechselwirkung geschlossener elektrischer Ströme auf die Wechselwirkung magnetischer Dipole zurückgeführt wird. Daraus folgert Hertz, daß für zeitlich veränderliche Magnetfelder («magnetische Ströme») ebenso wie für elektrische Ströme ein Vektorpotential angebbar ist.

Für den Zusammenhang zwischen Magnetfeld und Vektorpotential gilt nach Hertz folgender Zusammenhang:

mit

$$
\mathfrak{Z}_{1}=-\operatorname{rot} \mathfrak{U}_{1}
$$

$$
\operatorname{div} \mathfrak{U}_{1}=0 .
$$

Als Induktionsgesetz betrachtet Hertz die Gleichung

$$
\xi_{1}=-\frac{1}{\mathrm{c}} \frac{\partial}{\partial \mathrm{t}} \mathfrak{U}_{1},
$$

wobei das Vektorpotential und das Induktionsgesetz gegenüber der heute üblichen Schreibweise mit umgekehrtem Vorzeichen definiert sind.

In vollkommener Analogie zur Einführung eines Vektorpotentials für elektrische Ströme führt nun Hertz ein Vektorpotential (c für «magnetische Ströme» ein:

$$
\xi_{1}=\operatorname{rot} \xi_{1} .
$$

Vorausgesetzt ist dabei, was Hertz nicht ausdrücklich bemerkt, daß div $\leftleftarrows=0$ ist.

Entsprechend der Formulierung des Induktionsgesetzes für zeitlich veränderliche elektrische Ströme postuliert Hertz außerdem ein Induktionsgesetz für zeitlich veränderliche «magnetische Ströme»:

$$
\mathfrak{B}_{1}=-\frac{1}{\mathrm{c}} \frac{\partial}{\partial \mathrm{t}} \mathfrak{S}_{1} .
$$

Das Induktionsgesetz in Gleichung (20) hat also dieselbe Struktur wie das auf Franz E. Neumann zurückgehende Induktionsgesetz in Gleichung (18). Tritt im Falle der zeitlichen Änderung des Vektorpotentials elektrischer Ströme ein elek- 
trisches Feld auf, so bewirkt analog die zeitliche Änderung des Vektorpotentials «magnetischer Ströme» die «Induktion» eines magnetischen Feldes.

Bemerkenswert ist hier die Verwendung der Vektorpotentiale in den Gleichungen (18) und (20) im Sinne von Größen, deren zeitliche Ableitungen Meßgrößen sind. Dies entspricht allerdings dem historischen Stand der Theoriebildung. Auch Maxwell hatte weitgehend die Schreibweise von Gleichung (18) verwendet. Die heutige Vorstellung von der Mehrdeutigkeit, von der Nichtbeobachtbarkeit der Potentiale ist im Grunde erst durch die Arbeiten von Oliver Heaviside in die Elektrodynamik eingeführt worden.

Da die Gleichungen (17) bis (20) für Hertz noch keine vollständige Beschreibung zeitlich veränderlicher elektrischer und magnetischer Felder darstellten, machte er keinen Versuch, diese Differentialgleichungen direkt zu entkoppeln. Statt dessen führte er ein kompliziertes Näherungsverfahren (vgl. Anhang) durch, das im wesentlichen darauf beruht, daß ein physikalischer Vorgang angenommen wird, bei dem die Gleichungen (17), (18), (19) und (20) in dieser Reihenfolge realisiert werden $^{55}$ : Ein zeitlich veränderliches Magnetfeld $\mathfrak{K}_{1}$ bzw. ein zeitlich veränderliches Vektorpotential $\mathfrak{U}_{1}$ induziere also ein elektrisches Feld $\xi_{1}$. Dieses elektrische Feld sei ebenfalls zeitlich veränderlich, so daß ein zeitlich veränderliches Vektorpotential «magnetischer Ströme» resultiert. Dieses zeitlich veränderliche Vektorpotential $\complement_{1}$ bewirkt das Auftreten eines zusätzlichen Magnetfeldes, das zu dem anfänglich existierenden Magnetfeld addiert wird. Mit dem so «korrigierten » Magnetfeld wird dann der Zyklus erneut durchlaufen. Die Fortsetzung dieses Verfahrens und die Zusammenfassung der so entstehenden Reihen führt schließlich zu einer inhomogenen Wellengleichung für das Vektorpotential

$$
\Delta \mathfrak{Z}-\frac{1}{\mathbf{c}^{2}} \frac{\partial^{2}}{\partial \mathrm{t}^{2}} \mathfrak{A}=-4 \pi \dot{\mathrm{j}} \text {. }
$$

Entscheidend ist hier die durch das Näherungsverfahren erreichte Ergänzung der Poisson-Gleichung durch den Term $-\frac{1}{\mathbf{c}^{2}} \frac{\partial^{2}}{\partial \mathrm{t}^{2}} \mathfrak{U}$. Damit ist dasselbe Gleichungssystem wie das der Maxwellschen Gleichungen im ladungsfreien Fall erreicht. Mit den Gleichungen (17) und (18) ergibt sich aus der inhomogenen Wellengleichung (21)

$$
\operatorname{rot} \mathfrak{B}=-\frac{4 \pi}{\mathrm{c}} \mathrm{i}-\frac{1}{\mathrm{c}} \frac{\partial}{\partial \mathrm{t}}(\xi
$$

und mit dem heute üblichen Vorzeichen in Gleichung (17) und (18)

$$
\operatorname{rot} \mathfrak{B}=\frac{4 \pi}{\mathbf{c}} \mathrm{i}+\frac{1}{\mathrm{c}} \frac{\partial}{\partial \mathbf{t}}(\xi \text {. }
$$

d.h. das um den Vakuumverschiebungsstrom erweiterte Ampère-Gesetz. 
Nun stellt sich natürlich die Frage nach der Herkunft dieser zusätzlichen Information. Dann, wenn sie durch die Einführung des Vektorpotentials (5 für «magnetische Ströme» zustande kommt, ist zu fragen, ob die Ableitung konsistent ist. Wenn man zu diesem Zweck noch einmal die Ausgangsgleichungen (17) bis (20) betrachtet, ist es sinnvoll, hier die Hertzsche Auffassung der Unvollständigkeit fallenzulassen und die Gleichungen in der heute üblichen Weise zu entkoppeln. Wendet man auf Gleichung (20) Rotation an und setzt Gleichung (19) ein, erhält man

$$
\operatorname{rot} \mathfrak{B}=-\frac{1}{\mathrm{c}} \frac{\partial}{\partial \mathrm{t}} \mathbb{E} \text {. }
$$

Abgesehen vom Vorzeichen, ist dies die stromfreie Formulierung des AmpèreGesetzes, somit der Zusammenhang zwischen magnetischem Feld und Vakuumverschiebungsstrom. Geht man außerdem von der Formulierung des Induktionsgesetzes mit dem Vektorpotential über zu einer eindeutigen Formulierung mit dem Feld der magnetischen Induktion, so erhält man entsprechend der Vorzeichenwahl von Hertz

$$
\operatorname{rot} E=\frac{1}{c} \frac{\partial}{\partial t} \mathfrak{Z} \text {. }
$$

Nun ist nach der Voraussetzung div $\mathfrak{A}=0$ eine Coulomb-Eichung vorgenommen. Das Ampère-Gesetz in der Form (23), das Induktionsgesetz (24), die Beziehungen bzw. Voraussetzungen div $\mathfrak{Z}=0$ und $\operatorname{div} \xi=0$ liefern im Gegensatz zu Hertz' Ableitung keine inhomogene Wellengleichung, sondern wegen der CoulombEichung und der Eichung $\varphi=0$ die homogene Wellengleichung

$$
\Delta \mathfrak{U}-\frac{1}{c^{2}} \frac{\partial^{2}}{\partial t^{2}} \mathfrak{U}=0 \text {. }
$$

Auch an einem anderen Punkt erweist sich die Unstimmigkeit der Ableitung von Hertz. Betrachtet man noch einmal Gleichung (19) sowie das Ampère-Gesetz, das allerdings nur implizit vorausgesetzt wird, so ließe sich hier folgender Gedankengang anschließen: Analog zur Definition eines elektrischen Stromes mit Hilfe des Ampère-Gesetzes

$$
\operatorname{rot} \mathfrak{B}=-\frac{4 \pi}{c} \dot{i}
$$

wäre eine Definition des «magnetischen Stroms» $\dot{\mathrm{i}}_{\text {mag }}$ denkbar durch

$$
\operatorname{rot} \xi=\frac{4 \pi}{\mathrm{c}} \dot{\mathrm{I}}_{\text {mag }} \text {. }
$$

Dies würde aber andererseits zum Widerspruch mit div $(=0$ bzw. mit Gleichung (19) führen. 
Im Anschluß an die oben skizzierte Ableitung der inhomogenen Wellengleichung für das Vektorpotential hat Hertz die entsprechende Wellengleichung und die Maxwellschen Gleichungen bei Abwesenheit von Strömen und Ladungen angegeben. Die auffällige Symmetrie des Ampère-Gesetzes (23), des Induktionsgesetzes (24) und schließlich der Wellengleichungen für Felder sowie die von Hertz betonte Einheit von elektrischem Feld und magnetischem Feld und deren ungestörte Überlagerung haben zur Festigung der Maxwellschen Theorie beigetragen. Henri Poincaré $1891^{56}$ und noch 1903 Hendrik Antoon Lorentz ${ }^{57}$ haben sich in diesem Sinn über die Arbeit von Hertz (1884) geäußert, ohne auf die innere Problematik einzugehen ${ }^{58}$. Hertz hat seine Ableitung jedoch sehr zurückhaltend beurteilt :

«Ein solcher Beweis [der Maxwellschen Gleichungen] allein aus unseren Prämissen erscheint selbst unmöglich; das Genaue kann aus dem Ungenauen wohl als das von gewissem Standpunkte aus Nächstliegende, nicht aber als etwas Nothwendiges abgeleitet werden. $»^{59}$

1890 hat Hertz einen vollkommen anderen Weg eingeschlagen ${ }^{60}$, einmal dadurch, daß er wie Maxwell (bei den Wellengleichungen ${ }^{61}$ ) und (generell) wie Heaviside ${ }^{62}$ die Potentiale eliminierte, und zum andern dadurch, daß er die Theorie deduktiv aufbaute, so da $\beta$ die Maxwellschen Gleichungen, soweit sie voneinander unabhängig sind, einen axiomatischen Charakter erhielten. Das Leitmotiv der Theoriebildung der Hertzschen Arbeit von 1884 war eben nicht in erster Linie, einen direkten Beweis der Maxwellschen Gleichungen zu geben, sondern die Idee der Arbeiten von Riemann und Lorenz weiter zu vertiefen. Riemann und Lorenz hatten versucht, durch die Aufstellung von Wellengleichungen und durch die Angabe von retardierten Potentialen aus der Elektrodynamik heraus eine Theorie der Lichtausbreitung zu formulieren und damit gleichzeitig die ältere Elektrodynamik zu ergänzen. Hertz wollte aber nicht wie Riemann und Lorenz die inhomogene Wellengleichung für Potentiale als vollständige Theorie nur postulieren, sondern diese mit den Begriffen und Schlußweisen der älteren Elektrodynamik physikalisch begründen.

Anhang: Hertz' Ableitung der inhomogenen Wellengleichung für das Vektorpotential $\mathfrak{Z}$

Es bestehe ein Magnetfeld $\mathfrak{Z}_{1}$; also ist auch ein Vektorpotential $\mathfrak{Z}_{1}$ angebbar. Es sei nun durch Induktion ein elektrisches Feld $\xi_{1}$ tatsächlich erzeugt. Aus den Gleichungen (18) und (19) ergibt sich dann der Zusammenhang 


$$
\operatorname{rot} \mathfrak{C}_{1}=-\frac{1}{\mathrm{c}} \frac{\partial_{i} \mathfrak{A}_{1}}{\partial \mathrm{t}}
$$

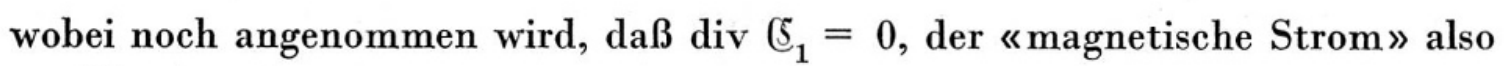
geschlossen sei.

Hertz wendet nun Rotation auf Gleichung (28) an und erhält

$$
\Delta \mathfrak{G}_{1}=\frac{1}{\mathrm{c}} \frac{\partial}{\partial \mathrm{t}} \operatorname{rot} \mathfrak{U}_{1} \text {. }
$$

Als Lösung dieser Differentialgleichung ergibt sich zunächst

$$
\mathfrak{S}_{1}=-\frac{1}{4 \pi \mathrm{c}} \frac{\partial}{\partial \mathrm{t}} \int \frac{\mathrm{rot}^{\prime} \mathfrak{U}_{1}\left(\mathrm{r}^{\prime}\right)}{\left|\mathrm{r}-\mathrm{r}^{\prime}\right|} \mathrm{d}^{3} \mathrm{r}^{\prime} .
$$

Durch partielle Integration läßt sich zeigen, daß dies identisch ist mit

$$
\mathfrak{G}_{1}=-\frac{1}{4 \pi \mathrm{c}} \frac{\partial}{\partial \mathrm{t}} \operatorname{rot} \int \frac{\mathfrak{U}_{1}\left(\mathrm{r}^{\prime}\right)}{\left|\mathrm{r}-\mathrm{r}^{\prime}\right|} \mathrm{d}^{3} \mathrm{r}^{\prime}
$$

In der Schreibweise von Hertz lautet die Lösung dann

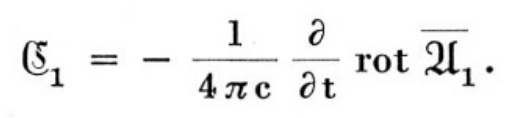

Die Gleichungen (17) bis (20) werden bei dem von Hertz gedachten physikalischen Vorgang innerhalb zeitlich veränderlicher magnetischer und elektrischer Felder in der Reihenfolge (17), (18), (19) und (20) realisiert. Man kann sich diesen Zyklus, den man im übrigen an jedem Punkt beginnen kann, durch folgendes Schema verdeutlichen:

$$
\mathfrak{Z}_{1}-(17) \rightarrow \mathfrak{U}_{1}-(18) \rightarrow \mathfrak{E}_{1}-(19) \rightarrow \mathfrak{E}_{1}-(20) \rightarrow \mathfrak{Z}_{\mathrm{k}}
$$

Das zusätzliche magnetische Feld $\mathfrak{Z}_{\mathrm{k}}$ ergänzt damit das ursprüngliche Feld $\mathfrak{Z}_{1}$ zu

$$
\mathfrak{Z}_{2}=\mathfrak{Z}_{1}+\mathfrak{Z}_{\mathrm{k}} \text {. }
$$

Damit kann der Zyklus von neuem durchlaufen werden:

$$
\mathfrak{B}_{2}-(17) \rightarrow \mathfrak{U}_{2}-(18) \rightarrow \ldots
$$

Um nun tatsächlich die Korrektur $\mathfrak{B}_{\mathrm{k}}$ zu formulieren, differenziert Hertz Gleichung (32) nach der Zeit und erhält

$$
-\frac{1}{\mathrm{c}} \frac{\partial}{\partial \mathrm{t}} \overleftarrow{\Xi}_{1}=\frac{1}{4 \pi \mathrm{c}^{2}} \frac{\partial^{2}}{\partial \mathrm{t}^{2}} \operatorname{rot} \overline{\mathfrak{U}}_{1} .
$$

Mit Gleichung (20) ergibt sich 


$$
\mathfrak{Z}_{\mathrm{k}}=\frac{1}{4 \pi \mathrm{c}^{2}} \frac{\partial^{2}}{\partial \mathrm{t}^{2}} \operatorname{rot} \overline{\mathfrak{Z}}_{1} .
$$

Damit ist eine erste Korrektur von $\mathfrak{Z}_{1}$ bzw. von Gleichung (17) gefunden, so daß Hertz

mit

$$
\mathfrak{B}_{2}=-\operatorname{rot} \mathfrak{U}_{2}
$$

$$
\mathfrak{U}_{2}=\mathfrak{U}_{1}-\frac{1}{4 \pi \mathrm{c}^{2}} \frac{\partial^{2}}{\partial \mathrm{t}^{2}} \overline{\mathfrak{U}_{1}}
$$

schreiben kann.

In der Gleichung (36) deutet sich bereits an, daß die Fortsetzung des Näherungsverfahrens von Hertz zu folgender Reihe für das vollständig korrigierte Vektorpotential $\mathfrak{Z}$ führt:

$$
\mathfrak{U}=\mathfrak{U}_{1}-\frac{1}{4 \pi \mathrm{c}^{2}} \frac{\partial^{2}}{\partial \mathrm{t}^{2}} \overline{\mathfrak{U}_{1}}+\frac{1}{16 \pi^{2} \mathrm{c}^{4}} \frac{\partial^{4}}{\partial \mathrm{t}^{4}} \overline{\overline{\mathfrak{U}_{1}}} \ldots
$$

Hertz wendet nun auf Gleichung (37) den Laplace-Operator an und erhält

$$
\Delta \mathfrak{Z}=-\frac{4 \pi}{\mathrm{c}} \mathrm{i}+\frac{1}{\mathrm{e}^{2}} \frac{\partial^{2}}{\partial \mathrm{t}^{2}} \mathfrak{U}_{1}-\frac{1}{4 \pi \mathrm{c}^{4}} \frac{\partial^{4}}{\partial \mathrm{t}^{4}} \overline{\mathfrak{U}_{1}} \ldots
$$

Dabei wird

$$
\Delta \mathfrak{U}_{1}=-\frac{4 \cdot \pi}{\mathbf{c}} \dot{\mathrm{i}}
$$

und (unter Berücksichtigung der Vorzeichenwahl von Hertz) letztlich auch

$$
\operatorname{rot} \mathfrak{B}_{1}=-\frac{4 \pi}{\mathrm{c}} \dot{\mathrm{i}}
$$

vorausgesetzt. Obwohl Hertz dieses Ampère-Gesetz nicht angibt, wird also an dieser Stelle der elektrische Strom $\mathfrak{j}$ in die formelmäßige Ableitung eingeführt.

Außerdem wendet er auf Gleichung (37) den Operator $\frac{1}{c^{2}} \frac{\partial^{2}}{\partial t^{2}}$ an und erhält

$$
\frac{1}{\mathrm{c}^{2}} \frac{\partial^{2}}{\partial \mathrm{t}^{2}} \mathfrak{U}=\frac{1}{\mathrm{c}^{2}} \frac{\partial^{2}}{\partial \mathrm{t}^{2}} \mathfrak{U}_{1}-\frac{1}{4 \pi \mathrm{c}^{4}} \overline{\mathfrak{U}_{1}} \ldots
$$

Subtrahiert man Gleichung (40) von Gleichung (38), so ergibt sich schließlich als Ergebnis seiner Überlegungen

$$
\Delta \mathfrak{Z}-\frac{1}{\mathbf{c}^{2}} \frac{\partial^{2}}{\partial \mathrm{t}^{2}} \mathfrak{U}=-\frac{4 \pi}{\mathbf{c}} \mathrm{j} .
$$




\section{Anmerkungen}

1 Vgl. dazu: The Correspondence of Isaac Newton, Vol. III. Ed. H. W. Turnbull, Cambridge 1961. Newton to Bentley. Letter 406. 25 February 1692/3. S. 253-256. Hier S. 253. Vgl. auch: Alexandre Koyré, Von der geschlossenen Welt zum unendlichen Universum [1957], Frankfurt 1969, S.163.

2 André Marie Ampère, Recueil d'observations électrodynamiques, Paris 1822, S. 214, 215.

${ }^{3}$ Brief von Carl Friedrich Gauß an Wilhelm Weber vom 19. März 1845. In: Gauß, Werke, Bd. V [1867], ${ }^{2}$ Göttingen 1877, S. 627 bis 629 . Hier S. 629.

4 Brief von Weber an Gauß vom 31. März 1845. Niedersächsische Staats- und Universitätsbibliothek Göttingen. Briefe an Gauß. Nr. 31, 1845. Zitiert nach: Karl Heinrich Wiederkehr, Wilhelm Webers Stellung in der Entwicklung der Elektrizitätslehre, Diss. Hamburg 1960, S. 68.

5 Wilhelm Weber, Elektrodynamische Maassbestimmungen. Über ein allgemeines Grundgesetz der elektrischen Wirkungen. In: Abhandlungen bei Begründung der Königl. Sächs. Gesellschaft der Wissenschaften, Leipzig 1846, S. 211-378. Auch in: Ders., Werke, Bd. 3, Berlin 1893, S. 25-214. Hier S. 30, 213, 214.

6 Vgl. Anm. ${ }^{5}$.

7 Wilhelm Weber. Vorwort bei der Übergabe der Abhandlung: Elektrodynamische Maassbestimmungen, insbesondere Zurückführung der Stromintensitäts-Messungen auf mechanisches Maass [1855]. In: Ders., Werke, Bd. 3, Berlin 1893, S. 591-596. Hier S. 594.

8 Wilhelm Weber, Elektrodynamische Maassbestimmungen. Insbesondere über elektrische Schwingungen [1864]. In: Werke, Bd. 4, Berlin 1894, S. 105-241. Hier S.157. Vgl. auch: Gustav Kirchhoff, U̇ber die Bewegung der Elektricität in Drähten. In: Annalen der Physik, G. F. Bd.176 (1857), S.193-217.

9 Bernhard Riemann, Ein Beitrag zur Elektrodynamik. In: Gesammelte mathematische Werke, Leipzig 1892. Nachträge 1902. Reprographischer Nachdruck als: Collected works of Bernhard Riemann, New York 1953, S. 288-293. Hier S. 293. Vgl. zu den Umständen der Publikation auch a.a. O. S. 553: Aus einem Brief Riemanns an eine seiner Schwestern geht hervor, daß die Veröffentlichung auch unter dem Druck von Gerüchten erfolgte, nach denen Gauß, der bereits 1855 verstorben war, eine ähnliche Theorie aufgestellt habe. Die Gerüchte bezogen sich wohl auf den erwähnten Brief von Gauß an Weber vom Jahr 1845. Vgl. dazu Anm. ${ }^{3}$.

10 Bernhard Riemann, Ein Beitrag zur Elektrodynamik. In: Poggendorffs Annalen der Physik, Bd.131, G. F. Bd. 207 (1867), S. 237-243.

11 Vgl. Anm. ${ }^{9}$. A.a.O. S. 288.

12 Riemann-Nachlaß. Niedersächsische Staats- und Universitätsbibliothek Göttingen. Cod. Riemann Ms. 24. Hier etwa 40 nicht paginierte Blätter mit Fragmenten zur Abhandlung «Ein Beitrag zur Elektrodynamik» von 1858 bzw. 1867.

13 Vgl. Anm. ${ }^{12}$. A. a. O.

14 Vgl. Anm. ${ }^{9}$. A.a. O. S. 290.

15 Vgl. Anm. ${ }^{12}$. A. a. O.

16 Vgl. dazu: Rudolf Clausius, Über die von Gauß angeregte neue Auffassung der elektrodynamischen Erscheinungen. In: Poggendorffs Annalen der Physik, Bd.135, G.F. Bd.211 (1868), S. 606-621. Hier S. 612-618. 
17 Vgl. Anm. ${ }^{9}$. A. a. O. S. 290.

18 Alfred Liénard, Champ électrique et magnétique produit par une charge électrique concentrée en un point et animée d'un mouvement quelconque. In: L'éclairage électrique, Tome XVI (1898), S. 5-14. Hier S. 6.

19 Emil Wiechert, Elektrodynamische Elementargesetze. In: Archives néerlandaises des sciences exactes et naturelles, Série II, Tome V, La Haye 1900, S. 549-573. Hier S. 564, 565. Auch in: Annalen der Physik, 4. F. Bd.4 (1901), S. 667-689. Hier S. 682.

${ }^{20}$ Vgl. Anm. ${ }^{19}$. [1900] a.a. O. S. 562, 563. [1901] a.a. O. S. 680. Vgl. zur Anregung durch (eigene) Kathodenstrahl-Experimente: [1901] a.a.O. S. 673, 674.

${ }^{21}$ Bernhard Riemann, Schwere, Electricität und Magnetismus. Nach den Vorlesungen [1861] ... bearbeitet von Karl Hattendorff, Hannover 1876, S. 329, 330.

${ }^{22}$ Vgl. Anm. ${ }^{21}$. A.a.O. S. 220, 221.

${ }^{23}$ Ludvig Valentin Lorenz, Über die Identität der Schwingungen des Lichts mit den elektrischen Strömen. In: Poggendorffs Annalen der Physik, Bd.131, G. F. Bd. 207 (1867), S. 243-263.

${ }^{24}$ Vgl. Anm. ${ }^{23}$. A.a. O. S. 243.

${ }^{25}$ Vgl. Anm. ${ }^{23}$. A.a. O. S. 244. - Nach Mogens Pihl ist jedoch kein weitergehender Einfluß von Oersted auf Lorenz nachweisbar. Vgl. dazu: Mogens Pihl, Der Physiker L.[udvig] V.[alentin] Lorenz, Kopenhagen 1939, S. 42.

26 Vgl. Anm. ${ }^{23}$. A.a. O. S. 261, 262.

27 James Clerk Maxwell, A treatise on electricity and magnetism, Vol. 2, Oxford 1873, S. 398, $\S 805$, Note; S. $435, \S 862$. Ders., On a method of making a direct comparison of electrostatic with electromagnetic force, with a note on the electromagnetic theory of light [1868]. In: Ders., The scientific papers, Vol. 2, Cambridge 1890, S.125-143. Hier S.137.

${ }^{28}$ Gustav Kirchhoff, Über die Bewegung der Elektricität in Leitern. In: Poggendorffs Annalen der Physik, Bd.102, G. F. Bd.178 (1857), S. 529-544. Hier S. 530.

29 Vgl. Anm. ${ }^{23}$. A. a. O. S. 247.

${ }^{30}$ Ludvig Valentin Lorenz, Mémoire sur la théorie de l'élasticité des corps homogènes à élasticité constante. In: Crelles Journal für die reine und angewandte Mathematik 58 (1861), S. 329-351. Hier S. 330, 331. Vgl. dazu auch: Mogens Pihl, Der Physiker L. V.Lorenz, Kopenhagen 1939, S.107-110.

${ }^{31}$ Vgl. Anm. ${ }^{23}$. A.a. O. S. 251. Vgl. dazu auch: Mogens Pihl, The Scientific Achievements of L. V.Lorenz. In: Centaurus 17 (1972), S. 83-94.

${ }^{32}$ Vgl. Anm. ${ }^{23}$. A.a. O. S. 251, 254.

${ }^{33}$ Ludvig Valentin Lorenz, Über die Theorie des Lichts. In: Poggendorffs Annalen der Physik, Bd.121, G. F. Bd. 197 (1864), S. 579-600. Hier S. 585.

${ }^{34}$ Vgl. Anm. ${ }^{23}$. A.a. O. S. 254, 255.

35 Vgl. Anm. ${ }^{23}$. A.a.O. S. 253.

36 Enrico Betti, Sopra la elettrodinamica; nota di ... In: Il Nuovo Cimento 27 (1868), S. 402-407.

37 Carl Neumann, Die Principien der Elektrodynamik (= Gratulationsschrift der Tübinger Universität zum fünfzigjährigen Jubiläum der Bonner Universität vom Jahr 1868). Auch in: Mathematische Annalen 17 (1880), S. 400-434.

38 Vgl. Anm. ${ }^{36}$. A.a.O. S. 402. 
39 Rudolf Clausius, Über die von Gauß angeregte neue Auffassung der elektrodynamischen Erscheinungen. In: Poggendorffs Annalen der Physik, Bd.135, G. F. Bd. 211 (1868), S. 606-621.

${ }^{40}$ Vgl. Anm. ${ }^{36}$. A.a. O. S. 405, 406.

41 Carl Neumann, Notizen zu einer kürzlich erschienenen Schrift über die Principien der Elektrodynamik [Vgl. Anm. ${ }^{37}$ ]. In: Mathematische Annalen 1 (1869), S. 317-324. Hier S. 320 .

42 Vgl. Anm. ${ }^{37}$. A.a. O. S. 418.

43 Vgl. dazu: L. [ev] D. [avidovič] Landau und E. M. Lifschitz [Evgeny Mikhailovich Lifshitz], Klassische Feldtheorie, ${ }^{4}$ Berlin 1967, S.183-186.

44 Hans-Jürgen Treder, Helmholtzsche und relativistische Elektrodynamik. In: Gerlands Beiträge zur Geophysik 79 (1970), S. 401-420. Hier S. 412.

45 Charles G.[alton] Darwin, The dynamical motions of charged particles. In: Philosophical Magazine 39, Ser. 6 (1920), S. 537-551. Hier S. 543.

${ }^{46}$ Gregory Breit, The effect of retardation on the interaction of two electrons. In: The Physical Review 34, Ser. 2 (1929), S. 553-573. Hier S. 555. Im Einheitsvektor des Abstandes steht fälschlich eine Zeitableitung.

47 Hermann Helmholtz, Über die Gesetze der inconstanten elektrischen Ströme in körperlich ausgedehnten Leitern [1870]. In: Ders., Wissenschaftliche Abhandlungen, Bd.1, Leipzig 1882, S. 537-544. Hier S. 539, 540. Und: Ủber die Theorie der Elektrodynamik. 1.) Über die Bewegungsgleichungen der Elektricität für ruhende leitende Körper [1870]. A.a. O. S. 545-628. Hier S. 548, 549, 567.

48 Heinrich Hertz, Über die Beziehungen zwischen den Maxwell'schen elektrodynamischen Grundgleichungen und den Grundgleichungen der gegnerischen Elektrodynamik. In: Annalen der Physik, N. F. Bd. 23 (1884), S. 84-103. Auch in: Ders., Gesammelte Werke, Bd.1, Leipzig 1895, S. 295-314.

${ }^{49}$ Heinrich Hertz, Erinnerungen. Briefe. Tagebïcher. Hrsg. von Johanna Hertz, Leipzig 1927, S. 145-14.7.

50 Vgl. Anm. ${ }^{23}$. A.a.O. S. 248. Hier die entsprechende Argumentation von Lorenz.

51 Vgl. Anm. ${ }^{48}$. Ann. d. Phys. A. a. O. S. 99. Und: Ges. Werke, Bd.1, S. 310.

52 Es spiegelt sich hier die immer wieder diskutierte historische und logische Abhängigkeit der Ampèreschen Entdeckung der Wechselwirkung von Strömen von Oersteds Entdeckung der Wechselwirkung von Strömen und Magneten. Vgl. z. B.: Ampère. In: Franz Arago's sämmtliche Werke. Hrsg. von W.G. Hankel, Bd. 2, Leipzig 1854, S. 3-94. Hier S. 49,50 .

53 Vgl. Anm. ${ }^{48}$. Ann. d. Phys. A. a. O. S. 85. Und: Ges. Werke, Bd.1, S. 296.

54 Vgl. Anm. ${ }^{48}$. Ann. d. Phys. A. a. O. S. 86-89. Und: Ges. Werke, Bd.1, S. 297-299.

${ }^{55}$ Vgl. Anm. ${ }^{48}$. Ann. d. Phys. A. a. O. S. 92 ff. Und: Ges. Werke, Bd. 1, S. $302 \mathrm{ff}$.

56 Henri Poincaré, Electricité et optique, Bd. 2, Paris 1891, S.114 ff.

57 Vgl. dazu: Hendrik Antoon Lorentz, Maxwells elektromagnetische Theorie [1903]. In: Encyklopädie der mathematischen Wissenschaften, Bd. 5, Teil 2, Leipzig 1904-1922, S. 63-144. Hier S. 99.

${ }^{58}$ Die direkt anschließende Diskussion der Hertzschen Arbeit betraf hauptsächlich das Webersche Grundgesetz : Eduard Aulinger, Über das Verhältniss der Weber'schen Theorie der Electrodynamik zu dem von Hertz aufgestellten Princip der Einheit der electrischen 
Kräfte. In: Annalen der Physik, N. F. Bd. 27 (1886), S.119-132. Hermann Lorberg, Bemerkungen zu zwei Aufsätzen von Hertz und Aulinger über einen Gegenstand der Electrodynamik. A.a. O. S. 666-673. Ludwig Boltzmann, Bemerkung zu dem Aufsatze des Hrn. Lorberg über einen Gegenstand der Electrodynamik. In: Annalen der Physik, N. F. Bd. 29 (1886), S. 598-603. Auch in: Ders., Wissenschaftliche Abhandlungen, Bd. 3, Leipzig 1909, S. 212-217. Vgl. dazu auch: Edmund T. Whittaker, A History of the Theories of Aether and Electricity, Vol.1, ${ }^{2}$ Edinburgh 1951, S. 321.

59 Vgl. Anm. ${ }^{48}$. Ann. d. Phys. A.a. O. S.102. Und: Ges. Werke, Bd.1, S. 313.

60 Heinrich Hertz, Über die Grundgleichungen der Electrodynamik für ruhende Körper. In: Annalen der Physik, N.F. Bd.40 (1890), S. 577-624. Hier z.B. S. 577, 578. Auch in: Ders., Gesammelte Werke, Bd. 2, Leipzig 1894, S. 208-225. Hier z.B. S. 208, 209. Interessant ist hierbei auch die Ansicht von Hertz, Maxwell sei von unmittelbarer Fernwirkung ausgegangen. Diese inhaltlich kaum nachzuweisende Behauptung ist einerseits Rechtfertigung der Neuformulierung der Maxwellschen Theorie, andererseits vielleicht ein gewisser Nachklang der Hertzschen Arbeit von 1884. Vgl. dazu auch: James Clerk Maxwell, Ủber Physikalische Kraftlinien. Hrsg. von Ludwig Boltzmann, Leipzig 1898 (= Ostwald's Klassiker der exakten Wissenschaften, Nr.102). Hier S. 85, 86: Anmerkungen Boltzmanns.

61 James Clerk Maxwell, A Dynamical Theory of the Electromagnetic Field. In: Philosophical Transactions of the Royal Society of London, Vol.155, Part I (1865), S.459-512. Hier S.498. Auch in: Ders., The Scientific Papers, Vol.1, S. 526-597. Hier S. 579. Und: Ders., On a Method of Making a Direct Comparison of Electrostatic with Electromagnetic Force; with a Note on the Electromagnetic Theory of Light. In: Philosophical Transactions of the Royal Society of London, Vol.158, Part II (1868), S. 643-657. Hier S. 655. Auch in: Ders., The Scientific Papers, Vol. 2, S.125-143. Hier S.141.

${ }^{62}$ Oliver Heaviside, On Electromagnetic Waves, § 1-14. In: Philosophical Magazine, 5. Ser., Vol. 25 (1888), S.130-156. Hier S.130, 131. Außerdem: Ders., Electromagnetic induction and its propagation. In: Ders., Electrical Papers, Boston 1925, Vol.1, S.429 bis 560 ; Vol. 2, S. $39-155$.

\section{Summary}

About 1865 James Clerk Maxwell had advanced his mathematical theory of the electromagnetic field. At the same time scientists on the continent still discussed a fundamental law of instantaneous interaction between electric charges or electric currents. On the other hand efforts were made within these theories to modify the principle of instantaneous action at a distance. So Bernhard Riemann (1858), Ludvig Valentin Lorenz (1867), Enrico Betti (1868) and Carl Neumann (1868) argued that the electrodynamical potentials are propagated in time. In this context Heinrich Hertz tried, in 1884, to "derive" Maxwell's Equations from the principles of theories of action at a distance.

Dr. Walter Kaiser

Fachbereich Mathematik der

Johannes-Gutenberg-Universität

Postfach 3980

D - 65 Mainz 